\title{
A List of Plants collected in Hang-chou, Cheh-kiang, by K. Honda. (Continued from Vol. XXVI. p. 346.)
}

\section{S. Matsuda.}

\section{Amarantaceæ.}

283. Achyranthes bidentata B1. ; Moq. in DC. Prodr. XIII. 2, 312 ; Hook. f. Fl. Brit. Ind. IV. 730 ; Wight Ic. Pl. Ind. Or. t. 779 ; Forb. et Hemsl. in Journ. Linn. Soc. XXVI. 322.

Ken-shan-mun (艮山門), Aug, (nos. 507,508) ; Tai-pin-mun (太本阴), Sept., (no. 427); Ching-tai-mun (清秦阴), Oct., (no. 514).

var. japonica Miq. (nom. Jap. Inokodzuchi) has somewhat smooth leaves, while in the type I notice the back of leaves. subvillose.

284. Alternanthera sessilis R. Br.; Moq. in DC. Prodr. XIII. 2, 357 ; Benth. Fl. Hongk. 286 ; Hook. f. Fl. Brit. Ind. v. 286 ; Wight. Ic. t. 727 ; Forb. et Hemsl. 1. c. 322 ; Diels in Engl. Bot. Jahrb. XXIX. 317.

Tsen-tang-inun (錢塘門), Oct., (no. 144).

Nom. Jap. Tsuru-nogeitō.

285 Amarantus paniculatus L ; Moq. in DC. Prodr. XIII. 2, p. 257 ; Hook f. Fl. Brit. Ind. IV. 718 ; Forb. et Hemsl. 1. c. 320 ; Diels 1. c. 316.

Ken-shan-mun (艮山門), Sept., (no. 11.); Oct., (no. 63).

Nom. Jap. Fujigeito.

Specim. no. 2 is an imperfect one.

285. A. spinosus L.; Moq. in DC. Prodr. XIII. 2, 260 ; 
Benth. Fl. Hongk. 284; Hook. f. Fl. Brit. Ind. IV. 718 ; Forb. et Hemsl. 1. c. 320 ; Diels 1. c. 316 ; Matsum. et Hayata, Enum. P1. Formos. 325.

Ku-shan (孤山), Oct., (no. 38)；Kon-yuan (貢院), Sept., (no. 584).

Nom. Jap. Hari-biyu.

Specim. no. 584 is an imperfect one.

286. Celosia argentea L., Moq. in DC. Prodr. XIII. 2, 242 ; Benth. F1. Hongk. 284 ; Forb. et. Hemsl. 1. c. 318 ; Diels 1. c. 316 .

Ya-feng (岳墳), Nov., (no. 830).

Nom. Jap. Nogeito (青葙).

\section{Chenopodiaceæ.}

287. Chenopodium album L. ; Moq. in DC. Prodr. XIII 2. 76 ; Benth. Fl. Hongk. 282 ; Hook. f. Fl. Brit. Ind. V. 3 ; Forb. et Hemsl. in Journ. Linn. Soc. XXVI. 323 ; Diels in Engl. Bot.. Jahrb. XXIX. 315.

Ken-shan-mun (艮山門), Oct., (nos. 422, 523); Chi-ling (葛嶺), Oct., (no. 308); Nov., (no. 154).

Nom. Jap. Akaza. (憼).

Chi-ling-specim. is too aged

288. Ch. ficifolium Smith; Moq. in DC. Prodr. XIII. 2, 65 ; Fr. et Sav. Enum. P1. Jap. I. 386 ; Forb. et Hemsl. 1. c. 324.

Ken-shan-mun (艮山門), Mai., (nos. 1300, 1301).

Nom. Jap. Ko-akaza.

289. Kochia Scoparia Schrad. Moq. in DC. Prodr. XIII. 2, 130 ; Hook. f. Fl. Brit. Ind. v. Il ; Fr. et Sav. Enum. Pl. Jap. I. 388 ; Horb. et Hemsl. 1. c. 428 ; Diels 1. c. 310.

Ken-shan-mun (艮山門), Nov., (no. 44?).

Nom. Jap. Hô-kigi (地膚).

\section{Phytolaccaceæ.}

290. Phytolacca esculenta van Houtte; Moq. in DC. 
Prodr. XIII. 2, 460; Walter in Engl. Pfl. Reich. Heft 39 (Phytolaccac.), 40.

Kong-yuan (貢院), Oct., (nos. 721, 815).

Nom. Jap. Yamagobō (商陸).

It differs from $P h$. acinosa. Roxb. thus: peduncles and axis of inflorescence are glabrous, and sepals obtuse in Ph. esculenta; sepals are acute, and peduncles and axis of inflorescence are scabrous in Ph. acinosa.

\section{Polygonaceæ.}

291. Polygonum Blumei Meisn. in Ann. Mus. Bot. Lugd. Bat. II. 57 ; Fr. et Sav. Enum. Pl. Jap. II. 473 et 479 ; Zōtei Somoku Zusetsu Vol. VII. Pl. 60 ; Nakai in Bot. Mag. Tokyo 1909, 394 (in Jap.) ; Lévl. in Bull. Soc. Bot. Fr. 1910, 449 ;= P. Posumbu Hamilt (Meisn). var. Blumei (Meisn). Herder; Nakai 1. c.

Tsen-tang-mun (錢塘門), Sept., (nos. 415, 416).

Nom. Jap. Inu-tade.

292. Polygonum (Sect. Cephalophilon) criopolitanum Hance in Ann. Sc. Nat. $5^{\text {me }}$ série, V. 238; Lévl. in Bull. Soc. Bot. Fr. (1910), 445. = P. capitatum Matsuda in Bot. Mag. Tokyo 1910, 172 non Hamilt.

Ken-shan-man (艮山門), Sept., (no. 322).

293. P. cuspidatum Sieb. et Zucc. Fl. Jap. Fam. Nat. n. 731 ; Meisn. in DC. Prodr. XIV. 136 ; Bot. Mag. t. 653 ; Forb. et Hemsl. in Journ. Linn. Soc. XXVI. 336 ; Diels in Engl. Bot. Jahrb. XXIX. 314.

Chi-ling. (葛嶺), Oct., (nos. 400, 498).

Nom. Jap. Itadori.

The present specimen has the margin of leaves crisped, and is closely allied to $P$. compactum Hook. f. in Bot. Mag. t. 6476.

294. P. Fagopyrum L. ; Baker et S. Moore in Journ. Linn. Soc. XVII. 385 ; DC. Orig. Pl. Cult. ed. 3, 279 ; Forb. et Hemsl. 1. c. $339 ;=$ Fagopyrum esculentum Moench.; Meisn. in DC. Prodr. XIV. 143 ; Hook. f. Fl. Brit. Ind. V. 55 ; Diels 1. c. 315. 
Tai-pin-mun (太本門), Oct., (nos. 690, 758, 759, 761, 762); Ching-tai-mun (清泰門), Mai., (no. 1299)； Aug., (nos. 374); Ken-shan-mun (艮山門), Oct., (no. 389).

Nom. Jap. Soba (薏货).

295. P. flaccidum Meisn.; Hook. f. Fl. Brit. Ind. V. 39; Forb. et Hemsl. 1. c. 339.

Ken-shan-man (艮山門), Oct., (no. 334); Tsen-tang-mun (錢垢門), Sept., (no. 36); Ku-shan (孤山), Nov., (no. 343).

Nom. Jap. Bontokutade.

This is P. fluccidum Meisn. in DC. Prodr. VIV. 109 in part. according to Hooker.

296. P. hangchouense n. s.

$8 \mathrm{dm}$. high, glabrous, branching; leaves shortly petiolate, ovato-oblong, acuminate, lamina $10 \times 1.5 \mathrm{~cm}$., glabrous above and underneath, punctated; stipules strigose, cilia shorter than the tube ; peduncles slender; racemes 3 or $4 \mathrm{~cm}$. long, subcylindrical, densely flowered; flowers whitish or slightly tinged purple (v. s.), fascicled in the axile of bracts, which are turbinate and furnished with short cilia; sepals $3 \mathrm{~mm}$. long; stamens 8 , unequal, included; style slightly exserted, branches 3 ; nut trigonose (mature one not seen).

This sp. has somewhat showy flowers, and is closely allied to P. Posumbu Hamilt. But short, densely flowered racemes, larger flowers, exserted style etc. distinguish it from the latter $\mathrm{sp}$. In the present sp. perianth about $3.5 \mathrm{~mm}$. long, style exserted, and stamens included; while in $P$. Posumbu I notice perianth not exceeding $2,5 \mathrm{~mm}$., style short, included, and the stamens subequal to the perianth.

Tai-pin-mun (太本門), Oct., (no. 684).

297. P. japonicum Meisn. in DC. Prodr. XIV. 112 et 695 ( $\beta$. grandiflorum Meisn.) ; Fr. et Sav. Enum. Pl. Jap. I. 396, et II. pp. 474 et 479 ; Forb. et Hemsl. 1. c. 341 ; Diels 312 ; Nakai in Bot. Mag. Tokyo (1909) 390 (in Jap.) ; Lévl. in Bull. Soc. Boe. Fr. (1910) 448.

Tsen-tang-mun (錢噳阿), Sept., (no. T02); Oct., (no. 411); Nov. (no. 371); Chi-ling (葛嶺), Oct., (no. 76); Ling-yin (靈鰠), Sept., (no. 379) ; Ken-shan-mun (艮山門), Sept. (nos. 7,465); 
Aug., (nos. 127,177); Ya-feng (岳填), Nov., (no. 453); Kü-shan (狐山), Aug., (no. 406).

Nom. Jap. Sakura-tade.

I notice styles 2 . in the specimen no. 702. In the no. 7 . sepals are about $3 \mathrm{~mm}$. long. Is this $P$. sterile Nakai 1. c. ?

298. P. lapathifolium L.; Hook. f. Fl. Brit. Ind. V. 35 ; Lévl. in Bull. Soc. Bot. Fr. (1910) 448;

var. incanum Ledeb. F1. Ross. III. 521; Matsuda in Bot. Mag. Tokyo XX. 145.

Yu-ching-mun (湧金門), Sept., (no. 147) ; Kong-tsen-chau (拱宸橋), Sept., (no. 540); Oct., (no. 549); Ken-shan-mun (艮山門), Oct., (nos. 779, 780).

Specim. 779 and 780 are somewhat deviating from the typical form, still they seem to be of the present sp., not of $P$. Persicaria L., which has hirsute and ciliate stipule.

299. P. multiflorum Thunb. F1. Jap. 169 ; Meisn. in DC. Prodr. XIV. 136 ; Hance in Journ. Bot. 1878, 13 et 1882, 293 ; Fr. et Sav. Enum. P1. Jap. I. 402, et II. 480 ; Diels 1. c. 314.

Ching-tai-mun (清泰門), Oct., (no. 44); Ku-shan (狐山), Oct., (no. 327).

Nom. Jap. Tsuru-dokudami (何首鳥).

300. P. nipponense Makino in Bot. Mag. Tokyo XXIII. 89 ; Nakai ibid. 420 (in Jap.); =P. hastato-sagittatum $\beta$. latifolium Makino in Bot. Mag. Tokyo XVII. $120 ;=P$. strigosum R. Br. var. latifolium Makino in litt. $;=P$. muricatum var. Maxim. in litt.

Tsen-tang-mun (錢䓵門), Sept., (nos. 30,32); Oct., (nos. 143, 337);

Ken-shan-mun (艮山門), Oct., (no. 461); Aug., (no. 409).

Nom. Jap. Yanone-gusa.

301. P. orientale L.; Meisn. in DC. Prodr. XIV. 123; Benth. Fl. Hongk. 288 ; Fr. et Sav. Enum. Pl. Jap. I. 397 et II. 478 ; Hook. f. Fl. Brit. Ind. V. 30 ; Bot. Mag. t. 213 ; Forb. et Hemsl. 1. c. 343 ; Diels 1. c. 313.

Ken-shan-mun (艮山門), Aug., (no. 496); Sept., (nos. 82, 83 ?); Oct., (nos. 81, 91, 500, 599 ); Tsen-tang-mum (錢塘門), Oct., (no. 420); Yu-ching-mun (湧金門), Nov., (no. 831). 
The determination of specim. no. 831 is unsatisfactory ; it seems to be a starved form of $P$. orientale.

Nom. Jap. Ôketade (葒草).

302. P. perfoliatum L. ; Forb. et Hems1. 1. c. 344; Diels 1. c. 314 .

Tai-pin-mun (太本門), Oct., (no. 404) ; Ken-shan-mun (艮山 門), Aug., (no. 412); Ching-tai-mun (清泰門), Oct., (no. 57).

Nom. Jap. Ishimikawa (杜板歸).

303. P. Posumbu Hamilt; Hook. f. Fl. Brit. Ind. v. 38 ; Fr. et Sav. Enum. P1. Jap. I. 394 et II. 473 ; Nakai in Bot. Mag. Tokyo, 1909, 394 (in Jap.); Lévl. in Bull. Soc. Bot. Fr. (1910). 449.

Ken-shan-mun (艮山門), Nov., (no. 356); Oct., (nos. 525, 601, 624); Sept., (no. 719); Aug., (nos. 515, 559); Swi-shingkau (水星閣), Nov., (no. 456); Tsen-tang-mun (錢塘門), Oct., (no. 412); Ching-tai-mun (清泰阴), Oct., (no.——); Tai-pin-mun (太本門), Oct., (no. 631).

Nom. Jap. Hana-tade.

Specim. 719 and 631 have short dense-flowered spike, and more than $1 \mathrm{fl}$. are in the axile of the bract.

304. P. virginianum L.; Meisn. in DC. Prodr. XIX. 112 ; Hook. f. Fl. Brit. Ind. v. 31 ; Forb. et Hemsl. 1. c. 352 ; Diels 1. c. 312 .

Ken-shan-mun (艮山門), Sept., (no. 6); Ku-shan (狐山), Oct., (no. 5).

Nom. Jap. Midzu-hiki.

forma glabratum $f$. $n$.

Leaves glabrous, not adpressedly haired.

Ku-shan (孤山), Oct., (no. 406).

305. Rumex acetosa L.; Meisn. in DC. Prodr. XIX. 63 ; Bak. et Moore in Journ. Linn. Soc. XVII. 385 ; Franch. P1. David. 253 ; Forb. et Hemsl. 1. c. 355 ; Diels 1. c. 311.

Tai-pin-mun (太平門), Apr., (nos. 955, 956); Ken-shan-mun (艮山門), Apr., (nos. 1063, 1064); Ku-shan (狐山), Mai., (no. 1033).

Nom. Jap. Suiba (酸模). 
306. R. crispus L.; Meisn. 1. c. 44; Benth. F1. Hongk. 286 ; Hance in Journ. Linn. Soc. XIII. 86 ; Fr. et Sav. Enum. Pl. Jap. I. 392 ; Forb. et Hemsl. 1. c. 356 ; Diels 1. c. $310 ;=$ $R$. japonicus Meisn. in Ann. Mus. Lugd. Bat. II. 56 ; Fr. et Sav. 1. c. (sec. Hemsley).

Mo-cha-bu (茅家埠), Mai., (no. 1334).

Nom. Jap. Gishi-gishi (羊蹻)

307. ? R. dentatus L.; Meisn. 1. c. 56 ; Hook. f. Fl. Brit. Ind. V. 59 ; Forb. et Hemsl. 1. c. 356 ; Diels 1. c. 311.

Bon-chan-mun (旺江門), Apr., (no. 115).

Fr. immature; determination unsatisfactory.

308. R. maritimus L.; Ledeb. Fl. Ross. III. 500 ; Meisn. 1. c. 56 ; Sow. Eng. Bot. VIII. 42. t. 1212 ; Hook. t. 1. c. 50 ; Thomé, F1. Deutsch1. II. 56, t. 193 A ; Forb. et Hemsl. 1. c. 357 ; Diels 1. c. 311 ; Makino in Bot. Mag. Tokyo (1905), 67.

Tai-pin-mun (太本門), Oct., (nos. 685, 686).

Nom. Jap. Kogane-gishigishi, Hama-gishigishi.

\section{Aristolochiaceæ.}

\section{Asarum. sp.}

Rhizome with short internodes, root fibrous, thick, leaf single, long petiolate (about $10 \mathrm{~cm}$ ), lamina cordato-reniform, obtuse or subacute $(6-7 \mathrm{~mm}$. broad) peduncle about $1 \mathrm{~cm}$. with membranaceous bract at base; flowers campanulate (12-15 $\mathrm{mm}$. high, $6 \mathrm{~mm}$. across), dark-purple?, smooth outside, elevatedly reticulate inside, 3-lobed, lobes ovate; filament short, anthers twice as long, anther-process very short; ovary half-superior ; styles or style-branches 6 , entire ?, obtuse, $2,5 \mathrm{~mm}$. long.

Mo-cha-bu (茅家埠), Apr., (nos. 1091, 1092).

Among the species in the key to the genus by Léveillé (Bull. Soc. Bot. Fr. Tom. 56, 609) the present sp. is not found.

\section{Piperaceæ.}

310. Houttuynia cordata Thunb. ; Fl. Jap. 234 t. 26 ; DC. Prodr. XVI. 1. 238 ; Benth. Fl. Hongk. 334; Hook. f. Fl. Brit. 
Ind. V. 78 ; Bot. Mag. t. 2731; Forb. et Hemsl. in Journ. Linn. Soc. XXXI. 364; Diels in Engl. Bot. Jahrb. XXIX. 272. Lu-po (二堡), Mai., (no. 1342-1344).

Nom. Jap. Dokudami (鬴荣).

311. Saururus Laureiri Decne.; C. DC. in DC. Prodr. XVI. 1, 239 ; Benth. et Hook. Gen. Pl. III. 128 ; Forb. et Hemsl. 1. c. 363 ; Diels 1. c. 272.

Chi-wang-shan (玉皇山), Jul., (no. 1357).

Nom. Jap. Hangeshō (三白草).

\section{Chloranthaceæ.}

312. Chloranthus Fortunei (A. Gr.) Solms in DC. Prodr. XVI. 1, 476 ; Forb. et Hemsl. in Journ. Linn. Soc. XXVI. 367 : Diels in Engl. Bot. Jahrb. XXIX. 273.

Tsu-yan-dong (紫雲洞), Apr., (no. 1013-1017, 1030.)

This sp. is distinct from Ch. japonicus Sieb. :

Median stamen fertile, connective $10 \mathrm{~mm}$. long or longer.

Median stamen sterile, connective 4 or $5 \mathrm{~mm}$. long.

Forbes's plant no. 1416 (from Feng-wang-shan) named $C h$. japonicus seems to be $\mathrm{C} h$. Fortunei.

\section{Lauraceæ.}

313. Cinnamomun Camphora Nees et Eberm.; Meisn. in DC. Prodr. XVI. 1, 24; Fr. et Sav. Enum. Pl. Jap. I. 411 ; Forb. et Hemsl. in Journ. Linn. Soc. XXVI. 371.

Tsen-tang-mun (錢塘門), Oct., (nos. 752, 753); Ken-shanmun (艮山門), Oct., (no. 851); Ku-shan (孤山), Mai., (nos. 1304?, 1369).

Nom. Jap. Kusunoki (樟).

314. Machilus Thunbergii Sieb. et Zucc. Fl. Jap. Fam. Nat. II. no. 704; Meisn. in DC. Prodr. XVI, 42 ; Miq. in Ann. Mus. Bot. Lugd.-Bat. II. 195; Fr. et Sav. Enum. P1. Jap. I. 411; Forb. et Hemsl. 1. c. 377 ; Banks, Icon. Kæmpf. t. 50 (fruct).

Pon-shan (牛山), Mai., (nos. 1335, 1336).

Nom. Jap. Inugusu. 


\section{Thymelæaceæ.}

315. Daphne Genkwa Sieb. et Zuce. Fl. Jap. I. 137, t. 75 ; Meisn. in DC. Prodr. XIV. 531; Fr. et Sav. Enum. Pl. Jap. I. 404; Forb. et Hemsl. in Jouru. Linn. Soc. XXVI. 395.

Tsu-yun-dong (紫雲洞), Apr., (no. 874); Chi-ling (葛嶺), Apr., (nos. 905, 907).

Nom. Jap. Fuji-modoki (艺花).

\section{Elæagnaceæ.}

316. ? Elæagnus multiflora Thunb. Fl. Jap. 66 ; Schlecht. in DC. Prodr. XIV. 614 ; Bot. Mag. t. 7341 ; Forb. et Hemsl. in Journ. Linn. Soc. XXVI. 404; Servettaz in Beih. Bot. Centralb. XXV. Abt. II. 58; =E. longipes A. Gr. Max. in Mél. Biol. VII. 560.

Ku-shan (孤山), Mai., (no, 1164).

Nom. Jap. Natsu-gumi?

Sterile specimen, determination unsatisfactory.

317. E. pungens Thunb. F1. Jap. 68; Schlecht. in DC. Prodr. XIV. 614; Max. in Mél. Biol. VII. 559 ; Fr. et Sav. Enum. Pl. Jap. I. 409 ; Forb. et Hemsl. in Journ. Linn. Soc. XXVI. 404 ; Servettaz 1. c. 77.

Ku-shan (孤山), Nov., (no. 345); Hwang-lung-dong (黄龍洞) Apr., (nos. 1082, 1084, 1090 ; fr).

Nom. Jap. Nawashiro-gumi.

318. E. sp.

Shrub; branches fuscous, more or less curving, leaves permanent, chartaceous, elliptic, obtuse, subapiculate, subcuneate at base, discolored, ashy-green and villose above, spotted with ferruginous dots underneath, (lamina 20-40 $8-17 \mathrm{~mm}$.); petiole 3-5 mm., canaliculate; flowers (not fully opened) autumnal, axillary, solitary, cernuous, ferrugiuous, fasciculate on the very short branches, perianth $9 \mathrm{~mm}$. long, densely haired both within without, tubular portian of the $\operatorname{limb}(3-4 \mathrm{~mm}$.), subprismatic, constricted below the lobes, attenuated towards the base and constricted there, lobes deltoid?, style smooth, disc not cons- 
picuous, pedicel $3-4 \mathrm{~mm}$. ferruginous.

This sp. seems to be allied to E. tonkinensis Servettaz, but differs from it in the leaves villose above. It may prove a new sp.

Ku-shan (孤山), Oct., (nos. 723, 724, 726).

\section{Santalaceæ.}

319. Thesium chinense Turcz.; DC. Prodr. XIV. 649; Hance in Journ. Bot. (1869) 42 ; Forb. et Hemsl. in Journ. Linn. Soc. XXVI. 408 ; Diels in Engl. Bot. Jahrb. XXIX. 306 ; $=T h$. decurrens Bl.; Fr. et Sav. Enum. Pl. Jap. I. 407.

Chi-ling (暮嶺), Apr., (no. 972).

Nom. Jap. Kanabikisō.

(to be continued.) 